\section{Sedimentary environments}

Ancient Sedimentary Environments. Second edition. By R. C. Selley. Pp. 287. (Chapman and Hall: London, 1978). Hardback £8; paperback £4.95.

THIS is an inexpensive book, in which the essential features of the major sedimentary environments are succinctly described. In a book of 287 pages, the treatment does not attempt to be detailed and each synthesis is supported by a generally well chosen list of references. The references, which form $17 \%$ of the text, are one of the strengths of the book, for they provide either the student or the experienced geologist with basic references up to 1977. Chapters cover, sedimentary environments and facies; river deposits; wind-blown sediments; lake deposits; deltas; linear clastic shorelines; mixed clastic; carbonate shorelines; shelf deposits; carbonate and terrigenous; reefs; flysch and turbidites; pelagic deposits, and sedimentary models, mythical and mathematical. As might be expected, the emphasis reflects the author's wide field experience, but the omission of glacial sediments is surprising.

The opening chapter contains definitions and discussions of a sedimentary environment and facies. Although brief, the discussion is a clear statement of important basic concepts. For instance-" One of the main problems of determining the origin of ancient sediments is that, though essentially reflecting depositional environments, they also inherit features of earlier erosional and non-erosional phases"-a truism which gives rise to many problems in interpretation.

The author points out that it is only sedimentary structures (and palaeocurrents) which unequivocally indicate the depositional environment. The five defining parameters of a sedimentary facies-geometry, lithology, sedimentary structures, palaeocurrents and fossils-are examined in an instructive way, flavoured with many cautions derived from experience.

In many cases, environmental interpretation must be based on borehole or seismic data, so the inclusion is very appropriate of a general section on the use of vertical grain-size profiles from geophysical logs and the use of the down-hole dipmeter.

The whole text is intended as a practical guide to "environmental interpretation [which] is at best an imprecise art, rather than a deterministic scientific discipline", though the author's treatment is a good preparation for travelling along the latter route. Generally, each chapter has a main section covering one or more case histories, followed by sections giving wider views of the particular sedimentary facies, its economic significance, subsurface recognition and finally a list of references. Numerous clearly presented diagrams reduce the need for lengthy descriptions.

By its very nature, the book is a compilation of generalisations, but these are made by a perceptive geologist who continuously reminds his readers that the exceptions are the

\section{Physiology of fungal colonies}

Practical Fungal Physiology. By P. M. Robinson. Pp. 122. (Wiley: Chichester, UK, and New York, 1978.) £3.50.

ONE can only agree with the author's opening statement in the preface to this book that "The potential of fungi as experimental material is not always fully exploited". This book provides a series of experiments designed to introduce a student to some basic aspects of fungal physiology. There are nine chapters and the suggested experiments are described in each chapter within an overall account of the cellular processes involved. Topics dealt with include spore germination, hyphal growth, fungal nutrition, morphogenetic substances, staling, colony morphology, mycostasis and continuous culture. The experiments described in most chapters are very basic and have been designed to utilise a minimum of expensive equipment. There are references to original papers in the relevant areas and figures are included to

\section{Molecular dynamics}

Molecular Structure and Dynamics. By W. H. Flygare. Pp. 696. (Prentice-Hall: Englewood Cliffs, New Jersey, and Hemel Hempstead, UK, 1978.) £18.25.

ONE can tell from the front leaf of the dustcover that this is a crowded treatise. There is the ${ }^{13} \mathrm{C}-\mathrm{NMR}$ spectrum of a methyl group with its free induction decay, the ESR spectrum of the cyclopentyl radical, the Raman spectrum of $\mathrm{CO}_{2}$, the low resolution IR spectrum of $\mathrm{CH}_{2} \mathrm{Cl}_{2}$, the radial wave function of the $\mathrm{I}^{-} 5$ s orbital, a coordinate system for electron scattering from a triatomic molecule and another relating laboratory and molecular axes, and the X-ray structure factor for really interesting problems. The whole presentation is admirably clear and concise, making it a most suitable book for a second-year university course Geologists at all levels will gain much information from the eminently readable and witty style, but the most important impact is the encouragement of a discriminating and critical sifting of geological evidence. At such an economical price, the book is a good investment.

D. Hamilton

D. Hamilton is Senior Lecturer in Creology at the University of Bristol, UK illustrate the expected results of certain experiments. Experiments are described in flowsheet form and adequate details are provided for media recipes, inoculation times and general experimental protocols.

Overall, this simplified approach should provide a reasonable introduction to the physiology of the fungal colony. It is, perhaps, unfortunate that many of the experiments involve aspects of fungal physiology of whose cellular and biochemical mechanisms we have little understanding. This limitation is compounded by the omission of experiments involving well understood phenomena such as the phototrophic responses of Phycomyces.

Despite these criticisms the book should prove useful to school and university teachers looking for ideas to be included in an introductory course on mycology. Also. if the book helps move the teaching of mycology away from the classical taxonomic approach. then it is to be welcomed.

Keith Gull

Keith Gull is Lecturer in Microbiology at the University of Kent, Canterbury, UK.

metallic potassium. If the back leaf had been used to show the electron density difference plot of $\mathrm{HF}$, the splitting pattern of a ${ }^{5} \mathrm{D}$ state in an octahedral field (with spin-orbit interaction), the Doppler spectrum of interstellar formaldehyde and the symmetry elements of $\mathrm{CH}_{4}$, then one could have dispensed with the statement of contents.

The book would be suitable for a BSc honours course in chemical physics or for a postgraduate course for spectroscopists. It is a well written book and I found little to quarrel with in either the contents or their presentation.

I must, however, admit to a preference for shorter books on a narrower front. Seven hundred pages is indigestible and I cannot envisage ever starting such a book and getting to the end. Of course, there are books on my shelf that I use just for reference, and per- 